editors have informed me that, if they themselves have any doubts about papers submitted for future issues of the journal, the manuscripts will also be examined by a second referee. Refereeing procedures, therefore, should be as tough as those employed by the more reputable journals.

Provided the determination of the editors to stop this journal becoming a depository for papers rejected elsewhere is maintained, and, if the editorial board continues to exercise a critical vigilance, there is every reason to hope that the standard of the first issue will be upheld.

I note with concern, however, that this is yet another specialist journal which now has to be perused. The proliferation of specialist periodicals during the last few years is a trend to be abhorred, partly because of their tendency to lower standards and partly because their very nature encourages narrow specialization which has a detrimental effect on the progression of science. Furthermore, in the present economic situation, libraries are being forced to become more and more selective in what they purchase and inevitably, if this trend continues, some journals must fail. A relatively expensive, specialist quarterly, such as Journal of Fluorine Chemistry, must surely be one of the most vulnerable.

J. H. Holloway

\section{School Physics}

PSSC Physics. By Uri Haber-Schaim, Judson B. Cross, John H. Dodge and James A. Walter. Third edition. Pp. 674. (Heath: Lexington, Massachusetts, and Farnborough, Hampshire, March 1971.) $£ 4$.

THIS is the third edition of a really most attractive elementary introduction to physics, a text which by now is firmly established in the United States. It is essentially aimed at a school leaving standard more or less corresponding to our A-level. Its 674 pages cover quite an extensive range, including optics, gravitation, energy, electrostatics, electromagnetics, elements of atomic structure, quanta and matter waves. The illustrations throughout the book are magnificent. The text is mainly descriptive, depending heavily on well drawn, and often amusing, illustrations, backed with a solid sprinkling of admirable photographs, some in colour and all very much to the point. The very backbone of the whole text is the many fine illustrations, for there is hardly a page but that it has one or more of these, all well drawn, cleverly designed and very informative.

There are perhaps one or two weaknesses in the text. The optics section is largely purely descriptive, at times a little superficial. Although, as elsewhere, the 160 pages devoted to optics have numerous delightful illustrations, yet there is, I think, a decided lack of derivation of elementary formulae, indeed not enough for O-level, much less A-level physics. However, when the reader moves straight on to the next sections (motion in a straight line and vectors) this defect is corrected. Especially clear and attractive are the sections on Newton's laws of motion and on gravitation, with just the right loading of mathematics for the A-level candidate. Since this quite essential amount of mathematics is correctly included here, all the more does one feel the weakness due to the paucity of simple mathematical treatments in the earlier fairly large section on optics. Much clever use is made of stroboscopic and interrupted photographs of moving objects to illustrate dynamical principles.

There follows a short section on the kinetic theory of gases which is excellent. There is a curiosity in the succeeding electrical section. It is perhaps a criticism that the description of the cathode ray tube and the use of the oscilloscope come even before the derivation of Coulomb's law in electrostatics. This seems to be giving in to modern popular expectations at the expense of a logical teaching sequence. A similar curiosity appears as early as page 5 of the text. Surely this is much too early to illustrate a large bevatron and even to write about "a common place Geiger counter". Such excitements should be left for later. This reminds me of a school I visited in the United States where boys were "familiar" with the use of a Geiger counter before they had been taught Ohm's law. Surely there is a very real educational danger in by-passing basic fundamentals early for the sake of modern (admittedly exciting) applications.

The sections of the text dealing with the magnetic field and electromagnetics are practical, well illustrated and backed up by just the right amount of mathematics. The chapters on the Rutherford atom, photons, atomic spectra and the wave theory of matter are all compact, adequate and treated with sympathetic understanding for the difficulties the schoolboy will encounter.

Each chapter has a set of problems and a book list for further reading. My only real criticism is that there is no section on acoustics, which lends itself admirably to the illustrative type of treatment used throughout this book. Production is first rate and the price reasonable, although high by school standards in this country. Yet this is a text of which there should certainly be more than one copy in every school library in this country. I welcome this new edition.

\section{Ionic Interactions}

Ionic Interactions: From Dilute Solutions to Fused Salts. Vol. 1: Equilibrium and Mass Transport. Edited by S. Petrucci. (Physical Chemistry: a Series of Monographs, Vol. 22.) Pp. xiii +407. (Academic: New York and London, February 1971.) \$19.50; $£ 9.10$.

THE word "Interactions" in the title must be read in nearly all of its connotations in order to connect the miniature monographs in this volume. It means the long range mutual electrostatic interactions of many ions in dilute solutions; it means the short range interactions which, depending on the forces and partners involved, lead to ionic association, solvation or complexation; in the second volume, interaction of ions with radiation will be included. Two types of interaction are omitted-chemical interactions of ions with other ions or molecules, and electrochemical interactions at the electrode-electrolyte (fused or solution) interface.

The first chapter, by $H$. Falkenhagen and W. Ebeling, presents a theoretical treatment of the equilibrium properties of dilute solutions of strong electrolytes. The classical limiting laws of Debye and Hückel for osmotic and activity coefficients are derived, first for point charges, and then for charged rigid spheres in a continuum. After a brief discussion of the limitations imposed by the linearized Poisson-Boltzmann equation, general molecular distribution functions are derived by the method of Bogoliubov; these lead to the thermodynamic properties as functions of concentration. Finally, ionic association as a consequence of electrostatic attraction is considered; the association constant is evaluated by requiring identity of corresponding coefficients in equations derived respectively for the physical model and the chemical model. The second chapter, by H. Falkenhagen, W. Ebeling and W. D. Kraft, summarizes the theory of electrolytic conductance as developed by the electrolyte school at Rostock University. First, the equation for the limiting tangent is derived, following the classical Debye-HückelOnsager approach. The authors then proceed to review their attack on the problem of irreversible processes in electrolytic solutions (conductance, in particular) which begins with a derivation of the fundamental equation of continuity from the exact Liouville equation of statistical mechanics. Solution of the former for the pair correlation function leads to the relaxation force. The electrophoretic effect is derived from the generalized diffusion equation of Falkenhagen and Ebeling. Combination of these two long range interionic effects then gives the conduc- 